in Proc. IEEE ICASSP-03, Hong Kong, April 2003, vol. IV, pp. 688-691

Copyright IEEE 2003

\title{
ADAPTIVE WIENER FILTERS FOR TIME-VARYING CHANNEL ESTIMATION IN WIRELESS OFDM SYSTEMS
}

\author{
Dieter Schafhuber, Gerald Matz, and Franz Hlawatsch \\ Institute of Communications and Radio-Frequency Engineering, Vienna University of Technology \\ Gusshausstrasse 25/389, 1040 Vienna, Austria (Europe) \\ phone: +4315880138973, fax: +4315880138999, email: Dieter.Schafhuber@ieee.org \\ web: http://www.nt.tuwien.ac.at/dspgroup/time.html
}

\begin{abstract}
In wireless OFDM systems, the time-varying channel is often estimated by a Wiener filter-type MMSE estimator based on pilot symbols. Such an estimator, however, requires statistical prior knowledge that is not easily obtained. Here, we propose adaptive Wiener filters for channel estimation that do not require statistical prior knowledge. We also calculate the performance limits of finite-length and infinite-length MMSE estimation. Simulation results demonstrate the good performance of our adaptive estimators.
\end{abstract}

\section{INTRODUCTION}

Orthogonal frequency division multiplexing (OFDM) [1] is an efficient modulation scheme for broadband wireless communications. To obtain high data rates, accurate channel estimation is required. A widely explored approach, minimum mean-square error (MMSE) channel estimation using Wiener-type filters [2-6], uses training data (pilot symbols) as well as knowledge of the channel's secondorder statistics. The latter requirement can be met by assuming some default channel statistics [5], explicitly estimating the channel statistics [6], or using a robust design approach [2-4].

Here, we propose adaptive Wiener filters for time-varying channel estimation that are attractive in that they do not require any statistical knowledge and are able to track changes of the channel statistics. We consider both the normalized least-mean-square (NLMS) and recursive least-squares (RLS) adaptation algorithms. As a basis for developing the adaptive estimators and as a reference for performance comparison, we also derive the finite-length and infinite-length MMSE channel estimators and calculate the performance limits for causal linear channel estimation. Related results for decision-directed channel prediction have been presented in [7].

The paper is organized as follows. After a review of the OFDM system in Section 2, the (nonadaptive) MMSE channel estimators are derived in Section 3. In Section 4, the adaptive estimators are presented. Finally, simulation results are shown in Section 5.

\section{OFDM SYSTEM MODEL}

We consider an OFDM system with $K$ subcarriers [1]. The transmit symbols are denoted as $a[n, k]$, where $n \in \mathbb{Z}$ is the OFDM symbol (time) index and $k \in\{0,1, \ldots, K-1\}$ is the subcarrier (frequency) index. The $a[n, k]$ are drawn from an arbitrary symbol constellation except at the pilot symbol locations $(n ; k) \in \mathscr{P}$ where $a[n, k]=p[n, k]$, with $p[n, k] \in\{-1,1\}$ being pilot symbols that are known to the receiver. The set of pilot locations is given by [8]

$$
\mathscr{P}=\{(n, k) \mid n \in \mathbb{Z}, k=i S+(n \bmod S), i \in[0, P-1]\} .
$$

Here, $P$ is the number of pilots per OFDM symbol and $S=K / P$ (assumed to be an integer) is the distance between adjacent pilots of an OFDM symbol. An example of these pilot locations is shown in

Funding by FWF grant P15156 and European IST project ANTIUM.

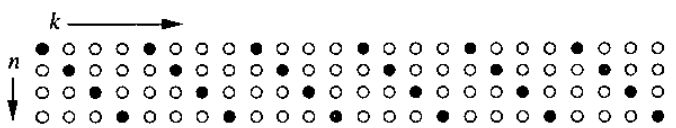

Figure 1: Location of pilot symbols (•) and data symbols (0) for $K=24, P=6$, and $S=4$.

Fig. 1. For later convenience, we define $p[n, k] \triangleq 0$ for $(n, k) \notin \mathscr{P}$. The $n$th OFDM symbol $s_{n}[m]$ is obtained by applying a normalized inverse discrete Fourier transform (IDFT) to the $a[n, k]$ and adding a cyclic prefix of length $L_{\mathrm{cp}}$,

$s_{n}[m]= \begin{cases}\frac{1}{\sqrt{K}} \sum_{k=0}^{K-1} a[n, k] e^{j 2 \pi m k / K}, & m=-L_{\mathrm{cp}},-L_{\mathrm{cp}}+1, \ldots, K-1 \\ 0, & \text { elsewhere. }\end{cases}$

Thus, each OFDM symbol has length $N=K+L_{\mathrm{cp}}$. The overall baseband transmit signal is $s[m]=\sum_{n=-\infty}^{\infty} s_{n}[m-n N]$.

Assuming a time-varying wireless channel with baseband impulse response $h[m, l]\left(l=0,1, \ldots, L-1\right.$, with $L \leq L_{\mathrm{cp}}+1$ the maximum delay) and additive noise $\eta[m]$, the received signal is given by

$$
r[m]=\sum_{l=0}^{L-1} h[m, l] s[m-l]+\eta[m] .
$$

The noise $\eta[m]$ is assumed white and Gaussian with variance $\sigma_{\eta}^{2}$.

The receiver discards the cyclic prefix and demodulates the received signal $r[m]$ by means of a normalized DFT, which yields

$$
x[n, k]=\frac{1}{\sqrt{K}} \sum_{m=0}^{K-1} r[n N+m] e^{-j 2 \pi k m / K} .
$$

If $h[m, l]$ varies negligibly within one symbol period, the input-output relation of the overall OFDM system is obtained as

$$
x[n, k]=H[n, k] a[n, k]+z[n, k],
$$

with the channel coefficients

$$
H[n, k]=\sum_{l=0}^{L-1} h_{l}[n] e^{-j 2 \pi k l / K}, \quad \text { where } h_{l}[n] \triangleq h[n N, l]
$$

and the noise $z[n, k]=\frac{1}{\sqrt{K}} \sum_{m=0}^{K-1} \eta[n N+m] e^{-j 2 \pi k m / K}$.

In what follows, the time-varying channel will be considered random. Under the wide-sense stationary uncorrelated scattering (WSSUS) assumption, $\mathrm{E}\left\{h\left[m^{\prime}, l\right] h^{*}\left[m^{\prime}-m, l^{\prime}\right]\right\}=r_{h}[m, l] \delta\left[l-l^{\prime}\right]$ $[9,10]$. The channel's scattering function is then defined as $[9,10]$

$$
S_{h}(l, v) \triangleq \sum_{m=-\infty}^{\infty} r_{h}[m, l] e^{-j 2 \pi v m}, \quad l=0,1, \ldots, L-1,
$$

with $v$ the normalized Doppler frequency. In what follows, $v_{\max }$ will denote the maximum Doppler frequency of the channel. The channel's path loss is defined as $\sigma_{h}^{2} \triangleq \sum_{l=0}^{L-1} \int_{-1 / 2}^{1 / 2} S_{h}(l, v) d v$. 


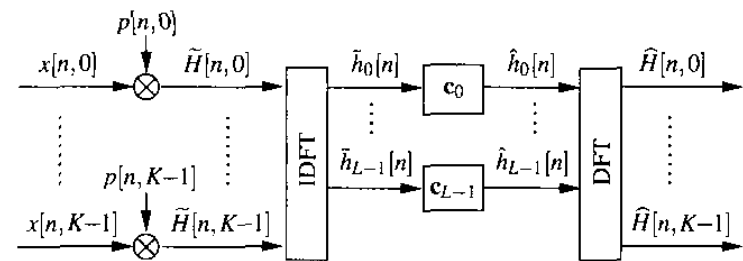

Figure 2: Block diagram of the channel estimator.

\section{WIENER FILTERS FOR CHANNEL ESTIMATION}

Fig. 2 shows the structure of the proposed MMSE channel estimator. (We note that related discussions of finite-length MMSE channel estimation were previously provided in $[2,3]$.) The estimator first multiplies the $x[n, k]$ received at the pilot locations by the pilot values. Using (1), we have

$$
\widetilde{H}[n, k] \triangleq x[n, k] p[n, k]= \begin{cases}H[n, k]+z \tilde{z}[n, k], & (n, k) \in \mathscr{P}, \\ 0, & \text { elsewhere }\end{cases}
$$

with $\tilde{z}[n, k]=z[n, k] p[n, k]$. In what follows, we assume $P \geq L$ to avoid aliasing. A scaled IDFT of the $\widetilde{H}[n, k]$ then yields

$\tilde{h}_{l}[n] \triangleq \frac{1}{P} \sum_{k=0}^{K-1} \widetilde{H}[n, k] e^{j 2 \pi k l / K}=h_{l}[n]+w_{l}[n], \quad l=0,1, \ldots, L-1$,

where $w_{l}[n]=\frac{1}{P} \sum_{k=0}^{K-1} \bar{z}[n, k] e^{j 2 \pi k l / K}$ is white noise with variance $\sigma_{w}^{2}=\sigma_{\eta}^{2} / P$. Note that for each $n$, this IDFT need only be calculated over the $P \leq K$ nonzero values of $\widetilde{H}[n, k]$.

Next, the channel impulse response is estimated using $L$ causal linear filters of length $M$, one for each channel tap:

$$
\hat{h}_{l}[n]=\sum_{m=0}^{M-1} c_{l}[m] \tilde{h}_{l}[n-m]=\mathbf{c}_{l}^{H} \tilde{\mathbf{h}}_{l}[n]
$$

with estimator coefficients $\mathbf{c}_{l}=\left[c_{l}^{*}[0] c_{l}^{*}[1] \ldots c_{l}^{*}[M-1]\right]^{T}$ and inputs $\tilde{\mathbf{h}}_{l}[n]=\left[\tilde{h}_{l}[n] \tilde{h}_{l}[n-1] \ldots \tilde{h}_{l}[n-M+1]\right]^{T}$. (Using $L$ separate scalar filters does not imply a loss of optimality because the channel taps are uncorrelated due to the WSSUS assumption.) We have $\tilde{\mathbf{h}}_{l}[n]=\mathbf{h}_{l}[n]+\mathbf{w}_{l}[n]$ with $\mathbf{h}_{l}[n]=\left[h_{l}[n] h_{l}[n-1] \ldots h_{l}[n-M+1]\right]^{T}$ and $\mathbf{w}_{l}[n]=\left[w_{l}[n] w_{l}[n-1] \ldots w_{l}[n-M+1]\right]^{T}$. Finally, the estimated channel coefficients are obtained as (cf. (2))

$$
\widehat{H}[n, k]=\sum_{l=0}^{L-1} \hat{h}_{l}[n] e^{-j 2 \pi l k / K}, \quad k=0,1, \ldots, K-1 .
$$

Finite-length MMSE channel estimator. By definition, the MMSE filter coefficients $\mathbf{c}_{l, \text { opt }}$ minimize the (normalized) MSE

$$
\varepsilon \triangleq \frac{1}{\sigma_{h}^{2}} \mathrm{E}\left\{|H[n, k]-\widehat{H}[n, k]|^{2}\right\}=\frac{1}{\sigma_{h}^{2}} \sum_{l=0}^{L-1} \mathrm{E}\left\{\left|h_{l}[n]-\hat{h}_{l}[n]\right|^{2}\right\} .
$$

Due to the orthogonality principle [11], $\mathrm{E}\left\{\left(h_{l}[n]-\hat{h}_{l}[n]\right) \tilde{h}_{l}^{*}[n-\right.$ $m]\}=0$ for $m=0,1, \ldots, M-1$ and $l=0,1, \ldots, L-1$. Inserting (3) yields the Wiener-Hopf equations

$$
\begin{gathered}
r_{h}[n N, l]-\sum_{m=0}^{M-1} c_{l}[m]\left(r_{h}[(n-m) N, l]+\frac{\sigma_{\eta}^{2}}{P} \delta[n-m]\right)=0, \\
n=0,1, \ldots, M-1, l=0,1, \ldots, L-1 .
\end{gathered}
$$

The MMSE coefficients are thus obtained as

$$
\mathbf{c}_{l, \mathrm{opt}}=\left(\mathbf{R}_{h}[l]+\frac{\sigma_{\eta}^{2}}{P} \mathbf{I}\right)^{-1} \mathbf{r}_{h}[l], \quad l=0,1, \ldots, L-1,
$$

with the correlation vectors $\mathbf{r}_{h}[l]=\mathrm{E}\left\{\mathbf{h}_{l}[n] h_{l}^{*}[n]\right\}$ and the correlation matrices $\mathbf{R}_{h}[l]=\mathrm{E}\left\{\mathbf{h}_{i}[n] \mathbf{h}_{i}^{H}[n]\right\}$ of size $M \times M$. The $\mathbf{R}_{h}[l]$ are Toeplitz with first columns $\mathbf{r}_{h}[l]$. The MMSE is given by [11]

$$
\left.\varepsilon_{\min }^{(M)} \triangleq \varepsilon\right|_{\mathbf{c}_{l}=\mathbf{c}_{i, \mathrm{opt}}}=1-\frac{1}{\sigma_{h}^{2}} \sum_{l=0}^{L-1} \mathbf{c}_{l, \mathrm{opt}}^{H} \mathbf{r}_{h}[l] .
$$

Infinite-length MMSE channel estimator. The case of infinite filter length, $M \rightarrow \infty$, characterizes the ultimate performance limit of causal linear MMSE estimation. Here, (4) can be solved using the Wiener-Hopf technique [12, Ch. 7.4]. In addition to $L \leq P$ and $L \leq L_{\mathrm{cp}}$, we assume $v_{\max } \leq 1 / N$ to avoid aliasing. Then, Fourier transforming (4) to the Doppler domain $(n \rightarrow v)$, we obtain

$$
\frac{1}{N} S_{h}\left(l, \frac{v}{N}\right)-C_{l}(v)\left[\frac{1}{N} S_{h}\left(l, \frac{v}{N}\right)+\frac{\sigma_{\eta}^{2}}{P}\right]=G_{l}(v),
$$

where $C_{l}(v)=\sum_{n=0}^{\infty} C_{l}[n] e^{-j 2 \pi v n}$ and $G_{l}(v)$ is undetermined except that it is the Fourier transform of a strictly anticausal sequence (because (4) is 0 for $n \geq 0$ ). We now use the spectral factorization

$$
\frac{1}{N} S_{h}\left(l, \frac{v}{N}\right)+\frac{\sigma_{\eta}^{2}}{P}=\rho_{l} F_{l}(v) F_{l}^{*}(v)
$$

where $F_{l}(v)=\sum_{n=0}^{\infty} f_{l}[n] e^{-j 2 \pi v n}$ with $f_{l}[n]$ minimum phase and monic $\left(f_{l}[0]=1\right)$. From the monicity property, it follows that [12]

$$
\rho_{l}=\exp \left\{\int_{-1 / 2}^{1 / 2} \ln \left[\frac{1}{N} S_{h}\left(l, \frac{v}{N}\right)+\frac{\sigma_{\eta}^{2}}{P}\right] d v\right\} .
$$

The factorization (8) is guaranteed to exist because $\rho_{l}>-\infty$ due to $\sigma_{\eta}^{2}>0$, i.e., the Paley-Wiener condition is satisfied [12]. Inserting (8) into (7) and identifying the causal parts of both sides of the resulting equation yields the optimal filter transfer functions

$$
C_{l, \mathrm{opt}}(v)=1-\frac{\sigma_{\eta}^{2}}{P \rho_{l} F_{l}(v)}, \quad l=0,1, \ldots, L-1 .
$$

A Doppler-domain expression of the MMSE is obtained from (6),

$$
\begin{aligned}
\varepsilon_{\mathrm{min}}^{(\infty)} & =1-\frac{1}{N \sigma_{h}^{2}} \sum_{l=0}^{L-1} \int_{-1 / 2}^{1 / 2} C_{l, \mathrm{opt}}(v) S_{h}\left(l, \frac{v}{N}\right) d v \\
& =\frac{\sigma_{\eta}^{2}}{P \sigma_{h}^{2}} \sum_{l=0}^{L-1}\left[\int_{-1 / 2}^{1 / 2} F_{l}^{*}(v) d v-\frac{\sigma_{\eta}^{2}}{P \rho_{l}} \int_{-1 / 2}^{1 / 2} \frac{1}{F_{l}(v)} d v\right],
\end{aligned}
$$

where (8) and (9) have been used. Both integrals evaluate to 1 due to the monicity property, so that we finally obtain

$$
\varepsilon_{\min }^{(\infty)}=\frac{\sigma_{\eta}^{2}}{P \sigma_{h}^{2}}\left[L-\frac{\sigma_{\eta}^{2}}{P} \sum_{l=0}^{L-1} \frac{1}{\rho_{l}}\right] .
$$

For a flat scattering function, i.e., $S_{h}(l, v)=\sigma_{h}^{2} /\left(2 L v_{\max }\right)$ for $[0, L-1] \times\left[-v_{\max }, v_{\max }\right]$ and $S_{h}(l, v)=0$ elsewhere, this becomes

$$
\varepsilon_{\min }^{(\infty)}=\frac{L \sigma_{\eta}^{2}}{P \sigma_{h}^{2}}\left[1-\left(1+\frac{P \sigma_{h}^{2}}{2 L N v_{\max } \sigma_{\eta}^{2}}\right)^{-2 N v_{\max }}\right] .
$$

From this expression, we see that $\varepsilon_{\mathrm{min}}^{(\infty)}$ is small for small maximum delay $L$, small maximum Doppler shift $v_{\max }$, large number of pilots $P$, small symbol duration $N$, and large SNR parameter $\sigma_{h}^{2} / \sigma_{\eta}^{2}$. 


\section{ADAPTIVE WIENER FILTERS}

The design of the Wiener filter-type channel estimator according to (5) or (9) requires prior knowledge of the second-order statistics of channel and noise. Moreover, if these statistics change the estimator would have to be redesigned. To avoid these complications, we propose adaptive channel estimators based on the NLMS or RLS algorithm [11]. These estimators continually update their coefficients without requiring prior knowledge of channel or noise statistics.

The estimated channel taps are (cf. (3))

$$
\hat{h}_{l}[n]=\mathbf{c}_{l}^{H}[n] \tilde{\mathbf{h}}_{l}[n], \quad n \geq 0, l=0,1, \ldots, L_{\mathrm{cp}},
$$

with time-varying adaptive filter coefficients $\mathbf{c}_{i}[n]$. We use $L_{\mathrm{cp}}+1$ filters since we do not know the true maximum delay $L$ (however, we assume that $L \leq L_{\mathrm{cp}}+1$ because otherwise (1) would not be valid). The coefficients are updated as

$$
\mathbf{c}_{l}[n]=\mathbf{c}_{l}[n-1]+\mathbf{k}_{l}[n-1] e_{l}^{*}[n], \quad n \geq 1,
$$

where $\mathbf{k}_{l}[n]$ is the update gain vector that will be defined presently and $e_{l}[n]$ is the error sequence defined as

$$
e_{l}[n] \triangleq \tilde{h}_{l}[n]-\hat{h}_{l}[n-1], \quad n \geq 1 .
$$

Note that in (12) we use the noisy reference $\tilde{h}_{l}[n]=h_{l}[n]+w_{l}[n]$ because $h_{l}[n]$ is unavailable. As a consequence, we delay $\hat{h}_{l}[n]$ by one sample (i.e., we use $\hat{h}_{l}[n-1]$ in (12)). This makes the error performance surface $J\left(\mathbf{c}_{l}\right)=\mathrm{E}\left\{\left|e_{l}[n]\right|^{2} \mid \mathbf{c}_{l}[n]\right\}$ (cf. [11]) independent of $\sigma_{w}^{2}$, which results in better performance of the adaptive filters. This delay, in turn, requires that we use $\mathbf{k}_{l}[n-1]$ instead of $\mathbf{k}_{l}[n]$ in (11). For initialization of the coefficient update, we will use

$$
\mathbf{c}_{l}^{(1)}[0]=\left[\begin{array}{llll}
1 & 0 & \ldots & 0
\end{array}\right]^{T} \quad \text { or } \quad \mathbf{c}_{l}^{(2)}[0]=\left[\begin{array}{llll}
0 & 0 & \ldots & 0
\end{array}\right]^{T} .
$$

NLMS algorithm. For the NLMS algorithm, the gain vector in (11) is (cf. [11])

$$
\mathbf{k}_{l}^{\mathrm{LMS}}[n]=\frac{\mu}{\left\|\tilde{\mathbf{h}}_{l}[n]\right\|^{2}} \tilde{\mathbf{h}}_{l}[n], \quad n \geq 1,
$$

where $\mu$ is the adaptation constant and $\left\|\tilde{\mathbf{h}}_{[}[n]\right\|^{2}=\tilde{\mathbf{h}}_{i}^{H}[n] \tilde{\mathbf{h}}_{l}[n]$. Stable operation requires $0<\mu<2$. The choice of $\mu$ is a trade-off between convergence speed and excess MSE. We obtained good results with $\mu \approx 0.05$. We prefer the NLMS algorithm over the LMS algorithm because the choice of $\mu$ is simpler [11]. [11])

RLS algorithm. For the RLS algorithm, the gain vector is (cf.

$$
\mathbf{k}_{l}^{\mathrm{RLS}}[n]=\frac{\mathbf{P}_{l}[n-1] \tilde{\mathbf{h}}_{l}[n]}{\lambda+\tilde{\mathbf{h}}_{l}^{H}[n] \mathbf{P}_{l}[n-1] \tilde{\mathbf{h}}_{l}[n]}, \quad n \geq 1,
$$

where the forgetting factor $\lambda$ with $0 \ll \lambda \leq 1$ accounts for possible nonstationarity of $h_{l}[n]$ (we obtained good results for $\lambda=0.99$ ). Furthermore, $\mathbf{P}_{l}[n]$ is the inverse of the sample correlation matrix $\sum_{i=0}^{n} \lambda^{n-i} \tilde{\mathbf{h}}_{l}[i] \tilde{\mathbf{h}}_{l}^{H}[i]$, which can be calculated recursively as

$$
\mathbf{P}_{l}[n]=\frac{1}{\lambda}\left(\mathbf{I}-\mathbf{k}_{l}^{\mathrm{RLS}}[n] \tilde{\mathbf{h}}_{l}^{H}[n]\right) \mathbf{P}_{l}[n-1], \quad n \geq 1 .
$$

For initialization of the RLS recursion, we set

$$
\begin{aligned}
\mathbf{P}_{l}[0] & =\left(\tilde{\mathbf{h}}_{l}[0] \tilde{\mathbf{h}}_{l}^{H}[0]+\delta \mathbf{I}\right)^{-1}=\frac{1}{\delta}\left[\mathbf{I}-\frac{\tilde{\mathbf{h}}_{l}[0] \tilde{\mathbf{h}}_{l}^{H}[0]}{\|\tilde{\mathbf{h}}[0]\|^{2}+\delta}\right], \\
\mathbf{k}_{l}^{\mathrm{RLS}}[0] & =\mathbf{P}_{l}[0] \tilde{\mathbf{h}}_{l}[0]=\frac{1}{\|\tilde{\mathbf{h}}[0]\|^{2}+\delta} \tilde{\mathbf{h}}_{l}[0],
\end{aligned}
$$

with a small stabilization parameter $\delta>0$ (we chose $\delta=0.1$ ).

Computational complexity. In each symbol interval, the coefficient update using the NLMS algorithm and the RLS algorithm has

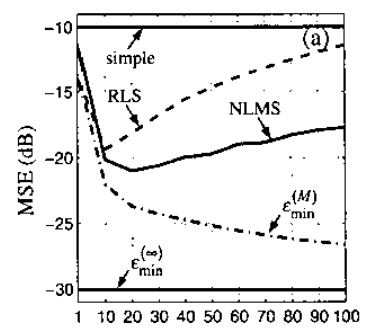

filter length $M$

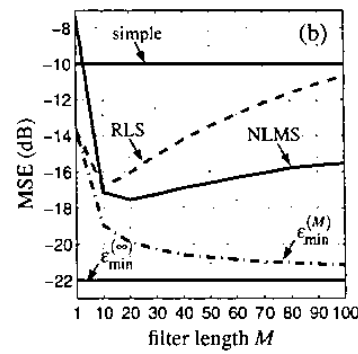

Figure 3: Channel estimation MSE vs. estimator filter length $M$ : (a) slow channel, (b) fast channel.

complexity of order $\mathscr{O}\left(M\left(L_{\mathrm{cp}}+1\right)\right)$ and $\mathscr{O}\left(M^{2}\left(L_{\mathrm{cp}}+1\right)\right)$, respectively. Hence, the RLS algorithm is significantly more complex. Furthermore, the computational complexity of the channel estimator in Fig. 2 is $\mathscr{O}\left(P \log _{2} P+M\left(L_{\mathrm{cp}}+1\right)\right)$ per symbol.

\section{SIMULATION RESULTS}

We simulated an OFDM system with $K=128$ subcarriers, symbol duration $N=144$, and cyclic prefix length $L_{\mathrm{cp}}=15$. Randomly chosen pilot symbols (with -1 and 1 equally likely) were transmitted on $P=16$ subcarriers; thus, $S=K / P=8$. The data symbols were taken from a 16-QAM constellation. The time-varying channel was simulated as described in [13], using a scattering function with Jakes Doppler profile [9] and exponential delay profile, i.e., $S_{h}(l, v)=\left(v_{\max }^{2}-v^{2}\right)^{-1 / 2} \exp \left(-l / \tau_{0}\right)$ with $\tau_{0}=L / \log _{e}(2 L)$ for $|v|<v_{\max }$ and $l=0, \ldots, L-1$ and $S_{h}(v, l)=0$ elsewhere.

We used $L_{\mathrm{cp}}+1=16$ adaptive filters (cf. Fig. 2) with NLMS adaptation constant $\mu=0.05$ and RLS forgetting factor $\lambda=0.99$. For comparison, we also simulated the nonadaptive maximum-likelihood channel estimator presented in [14] (hereafter briefly called "simple"), which uses the fixed coefficients $\mathbf{c}_{l}^{(1)}[n] \equiv\left[\begin{array}{llll}1 & 0 & \ldots & 0\end{array}\right]^{T}$, and we evaluated $\varepsilon_{\min }^{(M)}$ in (6) and $\varepsilon_{\min }^{(\infty)}$ in (10).

Performance vs. estimator filter length. First, we study the dependence of the MSE of the adaptive and nonadaptive channel estimators on the filter length $M$. We used $L=10$ and an SNR $\sigma_{h}^{2} / \sigma_{\eta}^{2}$ of $10 \mathrm{~dB}$. The MSE was estimated by averaging over $10^{4} \mathrm{OFDM}$ symbols. Figs. 3(a) and (b) show the results obtained for a slow channel $\left(v_{\max } K=0.001\right)$ and a fast channel $\left(v_{\max } K=0.01\right)$, respectively. It is seen that for growing $M, \varepsilon_{\min }^{(M)}$ decreases towards $\varepsilon_{\min }^{(\infty)}$; this decrease is quite rapid up to about $M=20$ but much slower afterwards. Thus, the dominant channel correlations can be exploited by a relatively short estimator filter. The MSE of the adaptive estimators (after convergence) decreases for $M$ up to about 10 (RLS) or 20 (NLMS). For $M=10$, the excess error of the adaptive estimators over $\varepsilon_{\min }^{(M)}$ is about $2 \mathrm{~dB}$. For higher $M$, the performance of the adaptive estimators degrades again since the excess error increases with the number of coefficients [11]. All Wiener filter-type estimators outperform the "simple" estimator.

Convergence of adaptive estimators. Fig. 4 shows the convergence of the adaptive channel estimators using the coefficient initializations $\mathbf{c}_{l}^{(1)}[0]$ and $\mathbf{c}_{l}^{(2)}[0]$ in (13). We chose the fast channel, an SNR of $10 \mathrm{~dB}$, and parameters $L=10$ and $M=10$. The MSE was estimated by averaging over 200 realizations. It is seen from Fig. 4(a) that initialization by $\mathbf{c}_{l}^{(1)}[0]$ results in a low initial MSE for both the NLMS and RLS algorithms (similar to the MSE of the "simple" estimator) but very slow convergence. Fig. 4(b) shows that 

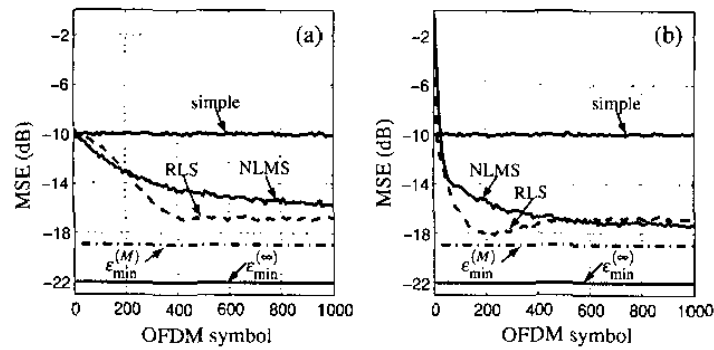

Figure 4: Convergence of the adaptive estimators: (a) initialization by $\mathbf{c}_{l}^{(1)}[0]$, (b) initialization by $\mathbf{c}_{l}^{(2)}[0]$.
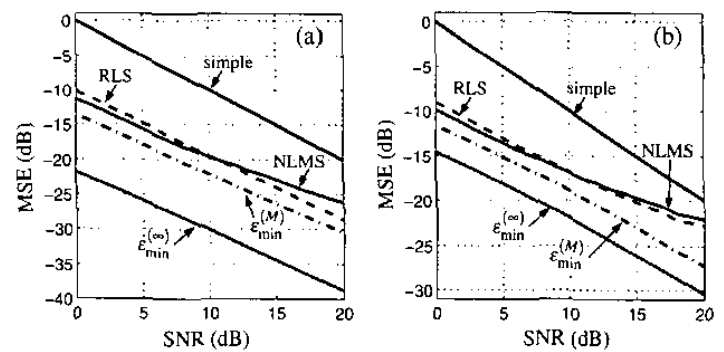

Figure 5: Channel estimation MSE vs. SNR: (a) slow channel, (b) fast channel.

using $\mathbf{c}_{l}^{(2)}[0]$, the initial MSE is high (about $0 \mathrm{~dB}$ ) but convergence is more rapid. The "simple" estimator is outperformed by the NLMS and RLS algorithm after 26 and 19 OFDM symbols, respectively.

Performance vs. SNR. Figs. 5(a) and (b) show the MSE vs. the SNR $\sigma_{h}^{2} / \sigma_{\eta}^{2}$ for the slow and the fast channel, respectively. We chose $L=10$ and $M=10$. The MSE was estimated by averaging over $10^{4}$ OFDM symbols. The excess error of the adaptive estimators over $\varepsilon_{\min }^{(M)}$ is seen to be about $3 \mathrm{~dB}$. Remarkably, the NLMS and RLS algorithms perform almost similarly, and they outperform the "simple" estimator by up to $10 \mathrm{~dB}$. For the fast channel, the excess MSE of the adaptive estimators and of $\varepsilon_{\min }^{(M)}$ over $\varepsilon_{\min }^{(\infty)}$ is smaller than for the slow channel. This is because the fast channel features less correlation in the time direction, which is sufficiently exploited already with $M=10$.

Variation of channel parameters. We finally analyze the dependence of the MSE on the channel parameters $L$ (maximum delay) and $v_{\max }$ (maximum Doppler). We used $M=10$, an SNR of $10 \mathrm{~dB}$, and averaging over $10^{4}$ OFDM symbols. Fig. 6(a) shows the MSE vs. $L$ for $v_{\max } K=0.01$ (fast channel). It is seen that the MSE increases as $L$ increases. Fig. 6(b) shows the MSE vs. $v_{\max } K$ for $L=10$. Here, the adaptive algorithms perform only about $3 \mathrm{~dB}$ worse than $\varepsilon_{\min }^{(M)}$ up to approximately $v_{\max } K=0.01$; beyond that, their performance degrades fast because the tracking error becomes dominant. The performance gap between $\varepsilon_{\min }^{(M)}$ and $\varepsilon_{\min }^{(\infty)}$ reduces with growing $v_{\max }$ due to the increasingly uncorrelated channel. From both parts of Fig. 6, it is again seen that the performance of the NLMS and RLS algorithms is very similar.

\section{CONCLUSIONS}

We developed adaptive Wiener filters for channel estimation in wireless OFDM systems. Simulations show that the performance of our adaptive estimators is only about $3 \mathrm{~dB}$ worse than that of the non-
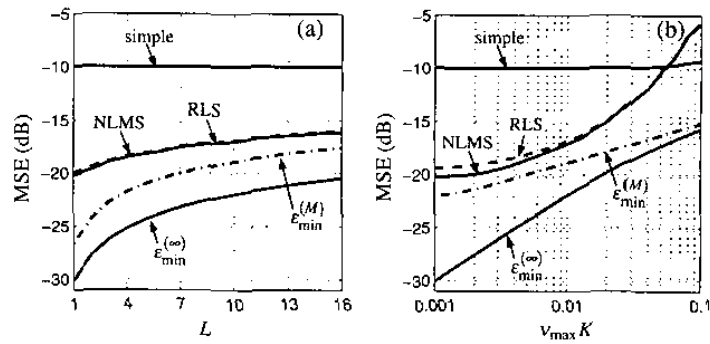

Figure 6: Channel estimation MSE vs. (a) maximum channel delay $L$, (b) maximum Doppler $v_{\text {max }}$.

adaptive Wiener filter (MMSE estimator) that requires knowledge of the second-order statistics of channel and noise. In our setup, the NLMS and RLS algorithms exhibit similar performance; thus, the simpler NLMS algorithm appears to be better suited for applications. We also derived a simple expression for the MSE of the infinite-length MMSE channel estimator and discussed its dependence on basic channel and system parameters.

\section{ACKNOWLEDGMENT}

The authors would like to thank M. Rupp for helpful discussions.

\section{REFERENCES}

[1] J. A. C. Bingham, "Multicarrier modulation for data transmission: An idea whose time has come," IEEE COmm. Mag., vol. 28, pp. 5-14, May 1990.

[2] Y. Li, L. Cimini, and N. Sollenberger, "Robust channel estimation for OFDM systems with rapid dispersive fading channels," IEEE Trans. Comm., vol. 46, pp. 902-915, July 1998.

[3] O. Edfors, M. Sandell, J.-J. van de Beek, S. K. Wilson, and P. O. Börjesson, "OFDM channel estimation by singular value decomposition," IEEE Trans. Comm., vol. 46, pp. 931-939, July 1998.

[4] Y. Li, "Pilot-symbol-aided channel estimation for OFDM in wireless systems," IEEE Trans. Veh. Technol., vol. 49, pp. 1207-1215, July 2000.

[5] P. Hoeher, "TCM on frequency-selective land-mobile fading channels," in Proc. S-th Tirrenia International Workshop, (Tirrenia, Italy), nels," in Proc. S-th Tirrenia
pp. 317-328, Sept. 1991.

[6] D. Schafhuber, G. Matz, F. Hlawatsch, and P. Loubaton, "MMSE estimation of time-varying channels for DVB-T systems with strong co-channel interference," in Proc. EUSIPCO-02, vol. III, (Toulouse, France), pp. 25-28, Sept. 2002.

[7] D. Schafhuber, G. Matz, and F. Hlawatsch, "Adaptive prediction of time-varying channels for coded OFDM systems," in Proc. IEEE ICASSP-2002, (Orlando, FL), pp. 2549-2552, May 2002.

[8] R. Negi and J. Cioffi, "Pilot tone selection for channel estimation in a mobile OFDM system," IEEE Trans. Consumer Electron., vol. 44, pp. 1122-1128, Aug. 1998.

[9] W. C. Jakes, Microwave Mobile Communications. New York: Wiley, 1974.

[10] P. A. Bello, "Characterization of randomly time-variant linear channels," IEEE Trans. Comm. Syst., vol. 11, pp. 360-393, 1963.

[11] S. Haykin, Adaptive Filter Theory. Englewood Cliffs (NJ): Prentice Hall, 1991 .

[12] T. Kailath, Linear Systems. Englewood Cliffs (NJ): Prentice Hall, 1980.

[13] D. Schafhuber, G. Matz, and F. Hlawatsch, "Simulation of wideband mobile radio channels using subsampled ARMA models and multistage interpolation," in 11th IEEE Workshop on Statistical Signal Processing, (Singapore), pp. 571-574, Aug. 2001.

[14] M. Morelli and U. Mengali, "A comparison of pilot-aided channel estimation methods for OFDM systems," IEEE Trans. Signal Processing, vol. 49, pp. 3065-3073, Dec. 2001. 